\title{
Hemispheric specialization in prefrontal cortex: effects of verbalizability, imageability and meaning
}

\author{
Daniel Casasanto
}

Department of Brain and Cognitive Sciences, Massachusetts Institute of Technology, 77 Massachusetts Avenue NE20-457, Cambridge, MA 02139, USA

\begin{abstract}
What determines the hemispheric laterality of information processing in the human prefrontal cortex (PFC)? Functional neuroimaging studies of declarative memory have explored two proposals: process-specificity and material-specificity. Early studies reported left-lateralized activation in prefrontal regions during memory encoding but right-lateralized activation during memory retrieval, for both verbal and nonverbal stimuli. Subsequent studies show that in addition to process-specific lateralization in some PFC regions, activation in other prefrontal regions varies according to the type of stimulus materials used. Verbal materials have been shown to preferentially activate regions of the left PFC and nonverbal materials homologous regions of the right PFC, during both encoding and retrieval. Findings are interpreted as consistent with the material-specificity hypothesis, which posits different lateralization of verbal and nonverbal memory processes [Hemispheric specialization and interaction, 1974]. Whereas initial fMRI and PET studies of material-specificity compared activation across material types (i.e. words vs. pictures) recent studies have begun to investigate hemispheric effects of stimulus manipulations within each material type. Based on these results and on findings in the behavioral and neuropsychological literature, it is hypothesized that activity in certain regions of left and right PFC may vary along two distinct, continuous dimensions: verbalizability and imageability. Recent experimental data will be evaluated according to the predictions of this proposal, and theoretical implications will be discussed.
\end{abstract}

(C) 2003 Elsevier Science Ltd. All rights reserved.

Keywords: Prefrontal cortex; Neuroimaging; Memory process

\section{Regional specialization in PFC}

Prefrontal cortex (PFC) is structurally and functionally heterogeneous, and no single theory of regional specialization can account for all available data. Most theories of arealization posit some combination of material-specificity and process-specificity, but

E-mail address: djc@mit.edu (D. Casasanto). 
these terms are used differently in different literatures to specify either intra-hemispheric or inter-hemispheric divisions of labor. ${ }^{1}$ In the working memory literature that has its roots in nonhuman primate electrophysiology, material-specificity is used interchangeably with domain-specificity, and commonly refers to the specialization of regions within ipsilateral dorsal and ventral PFC for spatial vs. nonspatial memory (Goldman-Rakic, 2000). In contrast, the process-specific view posits that dorsal and ventral PFC serve the same functions for both spatial and nonspatial stimuli, but are differentially engaged during maintenance and manipulation of information (Owen et al., 1998; Petrides, 1994). In the long-term declarative memory literature that has its roots in human neuropsychology, material-specificity refers to the specialization of left and right hemisphere structures for verbal and nonverbal memory, respectively (Milner, 1974; Wagner, 1998a). In contrast, one process-specific model asserts that left and right PFC are differentially engaged during episodic encoding and retrieval (Nyberg, Cabeza, \& Tulving, 1996; Tulving, Kapur, Craik, Moscovitch, \& Houle, 1994). Material-specific activation during declarative memory tasks is generally found in lateral posterior PFC regions, whereas process-specific activation tends to be found in more medial and anterior PFC, therefore materialspecificity and process-specificity may not be mutually exclusive (McDermott, Buckner, Petersen, Kelley, \& Sanders, 1999; Wagner, 1999). It is important to distinguish materialspecificity from modality-specificity (McCarthy \& Warrington, 1988), which posits localization of function based on sensory modality, and from category-specificity (Warrington \& Shallice, 1984), which posits separate loci in posterior neocortex for different semantic categories (e.g. animals vs. tools). Material-specific effects are not modality-specific, and have been found using aural/oral as well as visual/manual presentation and test modalities. Material-specific regions that respond differentially to words and pictures do not appear to discriminate semantic categories such as animals and artifacts that can be selectively impaired by damage to posterior cortex. This review will focus on the neuroimaging evidence for material-specific declarative memory processing in the lateral PFC. Studies will be selected from both the declarative memory and neurolinguistics literatures, as researchers with different theoretical motivations have used similar experimental paradigms. Recent findings will be evaluated according to the predictions of material-specificity, and an alternative account of the observed hemispheric variations will be argued, code-specificity.

\section{Neuropsychological evidence for material-specificity}

The material-specific model asserts that memory function lateralizes with language function: in left-language dominant individuals, the left hemisphere mediates verbal memory, and the right hemisphere nonverbal memory (Milner, 1974). The model is supported by numerous studies in temporal lobe epilepsy (TLE) patients who experienced selective memory deficits following unilateral resection of the epileptogenic medial temporal lobe (MTL). Removal of the left hippocampus and surrounding structures

\footnotetext{
${ }^{1}$ For an account of prefrontal function that emphasizes regional integration as opposed to segregation, see Miller and Cohen (2001).
} 
consistently produces verbal memory deficits, and although the findings are less robust, removal of the right hippocampal complex has resulted in memory deficits for nonverbal materials including mazes, unfamiliar faces, abstract patterns, and melodies (Blakemore \& Falconer, 1967; Jones-Gotman, 1986; Milner, 1968; Ojemann \& Dodrill, 1985; Plenger et al., 1996; Samson \& Zatorre, 1991).

The model has been extended to characterize lesion-deficit patterns in the frontal lobes. Unilateral frontal lobe lesions can produce material-specific deficits (Milner, 1982; Whitehouse, 1981), but such deficits are not always found. Left and right frontal lesion patients may perform indistinguishably on tasks for which left and right TLE patients show differential impairment (see Milner \& Petrides, 1984 for review; Petrides, 1985). Deficits following unilateral frontal insult differ in both severity and kind from those caused by MTL lesions. Whereas MTL lesions can produce profound deficits affecting multiple forms of declarative memory, frontal lesions tend to produce milder deficits, sparing recognition memory but impairing free recall and context-sensitive retrieval processes (Shimamura, 1995; cf. Wheeler, Stuss \& Tulving, 1995). Nevertheless, recent work by Kelley et al. (2002) confirms that the frontal lobes are important for forming new episodic memories, and provides strong evidence that their participation is materialspecific. Transient anesthesia of the left frontal lobe was found to selectively impair episodic memory for words, whereas transient anesthesia of the right frontal lobe impaired memory for unfamiliar faces in unilateral TLE patients.

\section{Neuroimaging evidence for material-specificity in PFC}

Several experiments on healthy subjects find differently lateralized activation for verbal and nonverbal stimuli during memory processing in the PFC, particularly in lateral and posterior regions. Wagner et al. (1998a) used fMRI to directly compare mnemonic processing of abstract words and chromatic visual textures, and found that words preferentially activated left inferior prefrontal cortex (LIPFC) and textures right inferior prefrontal cortex (RIPFC) during encoding and retrieval, thus providing one of the first demonstrations that prefrontal activation lateralizes according to stimulus type, as opposed to the stage of memory processing (i.e. encoding vs. retrieval). Similarly, McDermott et al. (1999) used fMRI to directly compare processing of object names and unfamiliar faces, and found that activation in the inferior frontal gyrus (IFG) (BA 6/44) was left-lateralized for words but right-lateralized for faces during both encoding and retrieval. In these studies, direct comparison of verbal and pictorial stimuli via subtraction analysis revealed regions that were preferentially activated by one type of stimulus, but not regions activated by both stimulus types. Kirchhoff, Wagner, Maril, and Stern (2000) compared novel words to repeated words and novel pictures to repeated pictures. Although both words and pictures elicited bilateral posterior PFC activation, novel words preferentially activated the posterior LIPFC (BA 6/44) whereas novel pictures preferentially activated posterior RIPFC (BA 6/44). Kelley et al. (1998) subtracted fMRI activation due to a low-level baseline task from activation during encoding of words, nameable line drawings, and unfamiliar faces. Regions of left lateral PFC were preferentially activated by words, and right lateral PFC by faces. 
Object drawings elicited nearly equivalent activation bilaterally in both right and left prefrontal cortices, in locations similar to those activated during word and face encoding.

\section{Hemispheric specialization within nonverbal materials}

The findings by Kelley et al. (1998) are of particular interest because they provide the first within-subject examination of hemispheric differences in functional activation not only across material types (words vs. pictures), but also within a material type (objects vs. faces). Objects and faces elicited comparable levels of right PFC activation. In contrast, objects, which are easier to verbally label than faces, showed dramatically greater left PFC activation. A similar pattern was found in the MTL. These finding are inconsistent with a simple prediction of differential left and right hemispheric involvement for verbal vs. nonverbal materials, but are nevertheless consistent with Brenda Milner's formulation of the material-specificity hypothesis. Milner wrote that whereas left hippocampal damage impairs "learning and retention of verbal materials", right hippocampal damage impairs memory for "complex visual and auditory patterns to which a name cannot be readily assigned" (Milner, 1974, p. 79, italics added). This implies that in addition to supporting memory for verbal materials, the left hippocampus also supports memory for nonverbal materials that are amenable to verbal description. If this pattern extends to the frontal lobes, then the left lateral posterior PFC region putatively specialized for verbal memory also mediates memory for nonverbal stimuli that are verbalizable. Activation in left lateral posterior PFC during nonverbal stimulus processing may be modulated by the ease with which the stimulus can be verbally coded or the extent to which verbal coding facilitates task performance. It is hypothesized that this activity reflects an internally generated mnemonic representation of the stimulus, and that left lateral posterior PFC activation varies with the extent to which the representation comprises verbal codes, irrespective of the external verbal or pictorial format of the stimulus. This proposal will be referred to as the verbalizability hypothesis.

A few neuroimaging studies have tested the verbalizability hypothesis, directly or indirectly. Golby et al. (2001) compared the hemispheric laterality of fMRI activation during encoding of verbal stimuli and three categories of nonverbal stimuli: abstract patterns, nonfamous faces, and complex scenes. Behavioral data collected prior to scanning suggested that these nonverbal stimulus types rely differentially on verbal processing during encoding. In a dual-task study, verbal interference affected recognition memory for faces and scenes more than recognition memory for abstract patterns. Imaging data reflected this difference, in both MTL and prefrontal regions of interest (ROIs). All four stimulus types elicited bilateral activation in the ROIs, but the laterality of activation differed across stimulus types. In the prefrontal ROI (BA 6), activation for novel vs. repeated stimuli was significantly left lateralized during word encoding, significantly right lateralized during pattern encoding, and nonsignificantly lateralized during face and scene encoding. Golby et al. provided compelling support for the present hypothesis by demonstrating verbalizability-correlated modulation of laterality across different categories of nonverbal stimuli. However, the strongest evidence would be hemispheric 
variation due to verbalizability within a single category of stimuli (e.g. faces, with and without associated verbalizable information). In a preliminary study, Kelley et al. (1999) reported bilateral frontal fMRI activation during famous but not nonfamous face processing, but this result has not been replicated elsewhere in the literature. Gorno-Tempini et al. (1998) showed no lateral prefrontal activation in their famous/ nonfamous face comparison. Simons, Graham, Owen, Patterson, and Hodges (2001) compared PET activation during retrieval of familiar and unfamiliar objects and faces. Face stimuli were photographs of famous or nonfamous people, and object stimuli were photographs of ordinary nameable objects or novel three-dimensional objects without names. Activation during all familiar stimuli (objects and faces combined) minus all unfamiliar stimuli yielded a single region of suprathreshold activation in the left ventrolateral PFC (BA 45), supporting the verbalizability hypothesis. The separate comparisons (familiar-novel objects and famous-nonfamous faces) did not yield any suprathreshold activation. Leveroni et al. (2000) compared fMRI activation during an old/ new recognition task for three types of faces: famous faces, nonfamous faces studied earlier in the experiment, and nonfamous faces that were previously unseen. They report bilateral frontal activity for famous faces relative to both studied nonfamous and previously unseen nonfamous faces, however this activation was found primarily in medial prefrontal loci. The one lateral prefrontal locus of activity correlating with famous face classification was right BA 47. This finding, which fails to support the verbalizability hypothesis cannot be discounted, but its interpretation is difficult. For all three types of face stimuli subjects pressed a button to indicate whether the face was previously seen or unseen, but 'previously seen' faces included both the famous faces (globally familiar, situationally unfamiliar) and the studied nonfamous faces (globally unfamiliar, situationally familiar). Deciding whether an unstudied famous face or a studied nonfamous face belonged in the previously seen category required resolving the conflict between global and situational familiarity. Famous faces were situationally novel. Since novelty has been shown to modulate the hemispheric laterality of activation during memory processing, promoting right hemisphere activation (Martin, 1999; Tulving, Markowitsch, Craik, Habib, \& Houle, 1996), it may be important to control situational familiarity across conditions. In a preliminary study, Casasanto, Kounios, and Detre (2001a) compared fMRI activation during encoding for blocks of nonfamous faces that varied in verbalizability, all of which were globally unfamiliar and situationally novel. The same set of university ID card photographs was prepared in two ways. For the first experiment, subjects viewed full head photographs, including the hair and neck. For the second experiment, a second group of subjects viewed the same set of face photographs, prepared differently. Each face photograph was cropped so as to include the brow, eyes, nose, mouth, and chin, but exclude ears, hair, and any clothing or jewelry. Subjects were instructed to remember the faces for a post-scan recognition test. Subjects reported using more verbal strategies during full head encoding than cropped face encoding, and postscan recognition memory performance was significantly better for full heads. Both cropped face encoding and full head encoding elicited bilateral activation relative to a scrambled face control task in a priori defined anatomical ROIs encompassing the inferior frontal gyri (IFG). A comparison of active suprathreshold voxels in the left and right IFG ROIs showed that activity was significantly right lateralized during cropped face encoding 
but significantly left lateralized during full head encoding. This finding supports the verbalizability hypothesis, but further studies are needed. The cropped face and full head photographs differed in more than just verbalizability; they also differed in visual complexity, and in some cases, the presence or absence of objects other than the face (e.g. shirt collar) which could be named and remembered independently. Ideally, the complexity of the nonverbal stimuli as well as their familiarity would be controlled across conditions.

In the preceding studies that support the verbalizability hypothesis, two differences between stimulus sets are confounded. Highly verbalizable stimuli such as familiar objects and famous faces also have pre-experimental meaning, whereas less verbalizable stimuli such as abstract patterns and unfamiliar faces have relatively little pre-experimental meaning. The observed activation in left PFC for verbalizable stimuli could correlate with verbal processing, per se, or with accessing stored semantic knowledge. Lexical processing and semantic retrieval have been localized to neighboring regions of the LIPFC (Fiez, 1997; Wagner, 1999; Wagner, Koutstaal, Maril, Schacter, \& Buckner, 2000). Since the exact location within the left lateral PFC for verbalizability-correlated activity varies across studies, it is not clear to what extent activation reflects verbal vs. semantic processing, a question which will be addressed in the final section of this paper.

\section{Hemispheric specialization within verbal materials}

If verbalizability modulates activity in left lateral PFC, what modulates activity in homologous right PFC regions? Activation in right posterior lateral PFC regions previously implicated in material-specific processing of nonverbal stimuli may vary according to the subject's internal representation of an item for both nonverbal and verbal stimuli. The degree to which the right lateral posterior PFC is engaged during verbal stimulus processing may be modulated either by the ease with which a stimulus can be mentally imaged, or the extent to which imagistic coding facilitates task performance. Right lateral posterior PFC activation is predicted to vary with the extent to which the internally generated mnemonic representation of a stimulus comprises imagistic codes, irrespective of the external verbal or pictorial format of the stimulus. This proposal will be referred to as the imageability hypothesis.

Casasanto et al. (2001b) investigated whether right hemisphere structures that have shown material-specific processing of nonverbal materials also support memory encoding for verbal materials that are highly imageable. FMRI activation was compared during encoding of concrete and abstract nouns. Imageability of stimuli correlated highly with concreteness $\left(r^{2}=0.81\right)$. Subjects reported generating more mental images of noun referents for concrete nouns than for abstract nouns during encoding, and post-scan recognition memory performance was significantly better for concrete than for abstract nouns. Both concrete and abstract nouns elicited bilateral activation during encoding relative to a consonant string control task in a priori defined anatomical ROIs encompassing the inferior frontal gyri. A comparison of active suprathreshold voxels in the left and right IFG ROIs showed that activity was significantly left lateralized during abstract noun encoding, but nonsignificantly 
lateralized (i.e. bilateral) during concrete noun encoding. This finding supports the imageability hypothesis, and is in agreement with several other studies in the neuroimaging literature. Kounios and Holcomb (1994) compared ERPs during categorization of concrete and abstract nouns, and found greater differences between concrete and abstract noun potentials over right anterior cortical sites than left anterior cortical sites. Friederici, Opitz, and von Cramon (2000) used fMRI to examine differences in neural activity due to word concreteness (abstract/concrete), word class (content/function), and task (syntactic/semantic). The semantic judgment task revealed bilateral frontal activity for both abstract and concrete content words, which appears to be left lateralized for abstract but not concrete words. Abstract content words activated three regions of left PFC (BAs 44, 44/6, 45) and right IFG, whereas concrete content words activated only one region of left PFC (BA 44/6) and right IFG. Z-scores were greater for abstract than for concrete content words in the left PFC, and greater for concrete than for abstract content words in the right PFC, a pattern which was confirmed in ROI analyses. Although this pattern supports the imageability hypothesis, no analysis was conducted to determine the significance of the hemispheric asymmetries in these regions. Fletcher, Shallice, Frith, Frackowiak, and Dolan (1996) directly compared PET activation during retrieval of verbal paired associates that varied in imageability, and found activation in left but not right dorsolateral PFC correlating with low-imageable but not high-imageable word retrieval. No lateral prefrontal activity was reported for the comparison of highimageable to low-imageable word retrieval. Schmidt et al. (2002) compared PET activation during paired-associate memory for high and low imageability nouns, presented either visually or aurally. Conjunction analysis showed that irrespective of presentation modality, encoding of high imageability pairs activated bilateral prefrontal cortex relative to baseline (BA 46), whereas encoding of low imageability pairs activated only the left PFC (BA 46). During retrieval, left lateral PFC was active for both for high imageability pairs (BA 46) and low imageability pairs (BA 46). Right lateral PFC (BA 46) was activated relative to baseline during retrieval of low but not high imageability pairs. The encoding findings support the imageability hypothesis, but the retrieval findings do not.

The encoding studies reviewed show preferential right frontal activity during imageable word encoding (Casasanto et al., 2001b; Friederici et al., 2000; Kounios \& Holcomb, 1994; Schmidt et al., 2002). In contrast, the retrieval studies report no right lateral frontal activity during imageable word retrieval (Fletcher et al., 1996; Schmidt et al., 2002). The process of encoding may require accessing and maintaining stored information about the stimulus, including information about its form, which can be represented imagistically. The retrieval-for-encoding of imageable stimulus properties is particularly likely when subjects are required to make a concrete/abstract judgment, as in three of the four encoding studies reviewed. In contrast, retrieving a target word upon presentation of the paired associate probe word may not require accessing any stored information about the target other than its linkage to the probe in the experimental setting. If this is the case, then the imageability-correlated activity in right lateral PFC found during the encoding tasks should be expected, and the absence of such activity during the paired associate retrieval tasks should also be expected. 
Two encoding studies designed to compare the neural substrates of concrete and abstract noun processing failed to support the imageability hypothesis. Kiehl et al. (1999) found bilateral fMRI activation in inferior frontal and middle frontal gyri, which did not differ significantly for concrete and abstract words. Beauregard et al. (1997) found PET activation in bilateral IFG (BA 46) for abstract words, but only in left IFG (BA 46) for concrete word processing. There are important differences between these encoding studies and those previously reviewed. Whereas the studies that support the imageability hypothesis encouraged deep processing of word stimuli, studies by Kiehl et al. and Beauregard et al. allowed shallow stimulus processing. Kiehl et al. (1999) asked subjects to make a lexical decision (word/nonword), and stated that "the participant was unaware of the concrete/abstract manipulation". In contrast, Casasanto et al. (2001b), Friederici et al. (2000), and Kounios and Holcomb (1994) required subjects to make concrete/abstract judgments. Kounios and Holcomb compared the results of lexical decision and semantic judgment experiments, and found the clearest hemispheric differences consistent with the imageability hypothesis when subjects performed a concrete/abstract noun judgment. It is possible that a lexical decision task does not engage the processes responsible for hemispheric effects found in other studies. Another reason why Kiehl and colleagues may have found little difference in brain activity between conditions (in PFC and other areas) is that their concrete and abstract blocks were composed of fifty percent nouns and fifty percent pronounceable pseudowords. Thus, activity correlated with pseudoword processing may have contributed to the results of their 'concrete stimuli vs. baseline' and 'abstract stimuli vs. baseline' comparisons as much as activity correlated with noun processing contributed to these results. Direct comparison of concrete vs. abstract blocks might have sidestepped this problem, but these comparisons did not yield any significant frontal activation. The study by Beauregard et al. not only allowed shallow processing of noun stimuli, but the experimenters actively discouraged deep processing, instructing subjects, "Do not think about the words (or letters) too much, just look at them as they appear on the screen." These instructions may account for their finding of no right PFC activation, if passive viewing of words fails to engage imagistic processing. Interpretation of the Beauregard et al. data is further complicated by the fact that this study failed to replicate the results of a similar concrete/abstract noun study by the same group (cited in Beauregard et al., 1997 as Chertkow et al., in press) which showed left IFG activation during abstract but not concrete noun processing: the opposite of the pattern reported in Beauregard et al. (1997).

One apparent limitation of the imageability hypothesis is that nonverbal materials may also be nonvisual. In addition to impairing visuospatial learning, right hemisphere lesions also impair learning of tactual mazes (Corkin, 1965) and musical melodies (Samson \& Zatorre, 1991). In the case of mazes, it is plausible that the tactual learning of spatial relationships results in a visually imageable mental map that aids in task performance in normal subjects but is compromised in patients with right hemisphere damage. Visual and spatial representations in working memory have been argued to depend upon the same neural structures in the right PFC (Nystrom et al., 2000; Owen et al., 1998; Postle, Stern, Rosen, \& Corkin, 2000; Smith \& Jonides, 1999). In the case of melodies, one possibility is that the right hemisphere subserves both visuospatial and musical representations, but that these representations are distinct. On this view, impairments for visuospatial learning and 
melody learning are unrelated, and the imageability hypothesis does not explain musical impairments. Alternatively, mental representations of music and space may be closely related. We talk about music using spatial terms: melody lines rise or fall; harmonies progress; notes are long or short, high or low. Music and space may share representational structure (Lakoff \& Johnson, 1980). PET data show that music and space share neural resources. The same regions in right dorsolateral and inferior PFC were engaged during attention to auditory stimuli that varied in either musical pitch or location (Zatorre, Mondor, \& Evans, 1999). On this view, the comorbidity of musical and visuospatial memory impairment is to be expected. A third possibility suggested by Whitehouse (1981) is that 'imagery' supported by the right hemisphere may not be limited to internal representations of visual experiences, but may also include mnemonic representations of experiences in other sensory modalities that 'isomorphically maintain significant features of the percept'. The role of the right PFC may be to sustain internally generated representations of haptic, auditory, and visual experiences which may all be considered imagistic, and which may share neural substrates whether or not they share representational structure.

\section{From material-specificity to code-specificity}

In PFC regions implicated in material-specific mnemonic processing, patterns of hemispheric specialization shown previously across material types have been demonstrated within verbal and nonverbal material types. Left lateral prefrontal regions show activation that correlates with the verbalizability of nonverbal stimuli (Casasanto et al., 2001a; Golby et al., 2001; cf. Gorno-Tempini et al., 1998; Kelley et al., 1998, 1999; Leveroni et al., 2000; Simons et al., 2001). Right lateral prefrontal regions show activation that correlates with the imageability of verbal stimuli (Beauregard et al., 1997; Casasanto et al., 2001b; Kiehl et al., 1999; Kounios \& Holcomb, 1994; Schmidt et al., 2002). It is hypothesized that activity in left lateral posterior PFC varies with the verbalizability of stimuli and activity in right lateral posterior PFC varies with the imageability of stimuli. This proposal, which combines the verbalizability and imageability hypotheses, will be referred to as code-specificity (McDermott et al., 1999; Paivio, 1971, 1991).

McDermott et al. (1999) also used the term 'code-specific' to describe their finding of differently lateralized activation during word and face encoding, but as it is implemented in their study, code-specificity differs from material-specificity only in name. McDermott et al. use 'material' and 'code' interchangeably in stating their hypothesis (see pp. 632633). The authors posit that code-specific activation "would presumably arise from the verbal/nonverbal processing demands afforded by the materials (and not the visual features of the materials themselves)," but they did not test this presumption. They neither demonstrated nor even hypothesized what is claimed presently: that both left lateralized verbal processing and right lateralized nonverbal processing can be evoked by either verbal or nonverbal stimulus materials. In fact, their study was incapable of testing the hypothesis that mnemonic code can dissociate from stimulus format, for two reasons. First, comparisons were made across but not within material types. Second, McDermott et al. compared activity during word and face encoding directly, thus their analysis was 
sensitive to areas differentially activated by words and faces but insensitive to regions activated by both stimulus types. The McDermott et al. study was important in that it further articulated the relationship between stimulus sensitivity and process sensitivity in the PFC, but it did not offer any new testable hypotheses regarding stimulus related hemispheric variation. Rather, McDermott et al. chose "to adopt the verbal/nonverbal heuristic... because that description has been common in the neuropsychological literature and seems to capture the current findings and predict other findings... reasonably well" ( $\mathrm{p}$. 637, italics added).

Code-specificity, as formulated presently, not only captures the McDermott et al. findings, it also accounts for an abundance of data which the 'verbal/nonverbal heuristic' cannot. Several behavioral studies on healthy subjects and lesion-deficit studies on frontal and MTL patients examined hemispheric specialization for verbal and nonverbal processing within a given stimulus category such as objects, faces, or nouns. Kaplan et al. (1994) found that when the right hemisphere was transiently anaesthetized, right TLE patients could verbally recall a visually presented object better than left TLE patients, suggesting that the left MTL mediates verbal aspects of visual object encoding. Seidenberg et al. (2002) found that left but not right TLE patients had difficulty naming famous faces, while performing like normal control subjects when asked to recognize famous faces and retrieve relevant semantic information. These studies show that the verbal processing associated with a nonverbal stimulus can be selectively impaired by left hemisphere damage.

Does the right hemisphere mediate imagistic coding of verbal materials? Seamon and Gazzaniga (1973) presented pairs of concrete nouns to both hemispheres in healthy subjects, with instructions to encode some pairs using verbal rehearsal and others using relational imagery (Bower, 1970). Probes were then shown in either the right or left visual hemifield. Test results showed an advantage for verbally rehearsed items when probes were presented to the left hemisphere (right visual hemifield), and an advantage for imaginally encoded items when probes were presented to the right hemisphere (left visual hemifield). Jones (1974) found that left TLE patients were severely impaired relative to normal controls on a paired associate recall task for concrete nouns, but that their performance improved significantly after they were trained to use relational imagery during noun encoding.

Can the nonverbal, imagistic processing associated with a verbal stimulus be selectively impaired by right hemisphere damage? Typically, performance dissociations for concrete and abstract words have been reported in aphasic patients with left hemisphere damage. Results of these studies are mixed. Some show that left hemisphere insult produces a selective deficit for abstract words; others show selective impairment for concrete words (see Saffran \& Sholl, 2000 for review). The more common finding is that concrete words are spared relative to abstract words following left hemisphere insult. While this is consistent with the hypothesis that the right hemisphere supports representations of imageable words, more compelling evidence is the selective impairment of concrete/imageable words following right hemisphere damage. Jones-Gotman and Milner (1978) trained right TLE patients and normal subjects to encode pairs of highly imageable words using relational imagery and pairs of low-imageable words by linking them together in a sentence. Patients performed like control subjects when required 
to retrieve low-imageable paired associates, but were significantly impaired when required to retrieve high-imageable paired associates. ${ }^{2}$ The severity of the impairment for highimageable word memory was positively correlated with the extent of right hippocampal excision.

Whereas the above patient-based studies concerned the MTL, Whitehouse (1981) directly tested both the verbalizability and imageability hypotheses in the frontal lobes. Patients with unilateral left or right anterior cerebral damage were tested on recognition memory for four types of stimuli: low imageability nouns, high imageability nouns, highly nameable pictures, and relatively nonnameable pictures. It was hypothesized that left hemisphere patients would show impaired verbal coding but intact imaginal coding whereas right hemisphere patients would show the opposite pattern, both between and within material types. Whitehouse posited that low imageability nouns and low verbalizability pictures are represented by single codes, whereas high imageability nouns and highly verbalizable pictures are dually codable, as argued by Paivio (1991). Earlier experiments suggest that the primary code by which a stimulus is represented reflects its presentation format (i.e. pictorial or verbal), and that some time is required to activate a secondary code for dually codable stimuli (Paivio \& Csapo, 1969). Thus, Whitehouse presented stimuli at two rates, hypothesizing that any performance advantage for dually codable stimuli would be more apparent at the slower rate than at the faster rate. In particular, it was predicted that patients with left hemisphere damage would benefit from the secondary imaginal code when encoding imageable nouns at slow rates more than at fast rates. Likewise, patients with right hemisphere damage would benefit from the secondary verbal code when encoding verbalizable pictures at slow rates more than at fast rates. These predictions were upheld by a significant three-way interaction (patient group $\times$ stimulus type $\times$ presentation rate). Results were supported by a second experiment showing differential effects of verbal and imaginal interference on memory performance in the left and right hemisphere patients.

Whitehouse (1981) showed that anterior right hemisphere damage impaired memory for imageable words more than nonimageable words, and anterior left hemisphere damage impaired memory for verbalizable pictures more than nonverbalizable pictures. These results show that verbal aspects of nonverbal stimulus encoding are mediated by the left hemisphere, and that nonverbal aspects of verbal stimulus encoding are mediated by the right hemisphere. This study provides the strongest, and most complete evidence to date in support of the code-specificity hypothesis. By adapting Whitehouse's design for a withinsubjects neuroimaging study, varying both the type of stimulus material and the codability of stimuli within each material type, it may be possible to demonstrate code-specific hemispheric specialization in PFC regions previously implicated in material-specific processing.

Why is it important to distinguish code-specificity from material-specificity? Materialspecificity does not commit to the level of mental representation at which the hypothesis is proposed to operate. To review, Milner's (1974) statement of material-specificity implies that the left hemisphere mediates memory for stimuli that are either verbal or verbalizable.

\footnotetext{
${ }^{2}$ The Schmidt et al. (2002) results suggest that relational imagery may have benefited normal subjects but not right TLE patients during encoding as opposed to retrieval, but the effect was revealed through retrieval testing.
} 
The symmetrical statement regarding the right hemisphere has not been made, perhaps because the first conclusive neuropsychological evidence was reported two decades after material-specificity was formulated. Nevertheless, Jones-Gotman and Milner's (1978) finding of impaired image-mediated verbal learning in right TLE patients suggests that the right hemisphere mediates memory for stimuli that are either nonverbal or imageable. ${ }^{3}$ Because of the 'or' in 'verbal or verbalizable' and 'nonverbal or imageable' it is unclear whether hemispheric specialization is hypothesized to depend upon an input driven representation of the stimulus or on internally generated representations: 'verbal/ nonverbal' implies the former, and 'verbalizable/imageable' the latter. Materialspecificity comprises two hypotheses that must be distinguished and evaluated separately: hemispheric specialization based on the verbal or nonverbal format of the stimulus (which might more accurately be called format-specificity), and hemispheric specialization based on the internally generated verbal or imagistic codes by which a stimulus is represented during memory processing, which has been called code-specificity.

The conflation of format-specificity and code-specificity may not be particularly problematic when the material-specific model is applied as originally intended, to characterize and predict memory impairments in TLE patients. At minimum, materialspecificity is a useful heuristic because of the relationship between the format in which a stimulus is presented and the codes by which it is represented in memory: all verbal materials are verbalizable, and all pictorial materials are imageable. Crucially, however, the converse is not true. The failure to distinguish material-specificity's component hypotheses, and therefore the failure to specify the level of mental representation at which 'material' is defined, has led to confusion in the neuroimaging literature.

'Material type' is generally identified with the external format of stimuli, not with internally generated representational codes, as is evident from the canonical description of material-specificity as a verbal/nonverbal distinction (not a verbalizable/imageable distinction). Further evidence that 'material type' is principally identified with format and not code is the fact that code-specific effects in the right hemisphere were not reported during the first twenty years of material-specificity research, were not predicted even by the original framer of the hypothesis, ${ }^{3}$ and do not appear to have been incorporated into any published formulation of material-specificity. Violations of format-specificity in neuroimaging results have sometimes been interpreted as violations of materialspecificity, but not always. For example, unfamiliar faces are considered a paradigm case of right hemisphere mediated stimuli, yet both Grady et al. (1995) and Haxby et al. (1996) found left lateralized activation during unfamiliar face encoding using PET, as did Casasanto et al. (2001a) using fMRI. The findings by Grady and Haxby have been repeatedly cited as exceptions to material-specificity, but an explanation has been offered in terms of code-specificity. Kelley et al. (1998) speculate that left hemisphere activity may have resulted from subjects' use of verbal encoding strategies promoted by long stimulus presentation times. On this account, code-specificity explains stimulus-related hemispheric effects, not format-specificity, which predicts the opposite result. The same explanation of verbal coding for nonverbal stimuli is given to account for left hemisphere

\footnotetext{
3 Jones-Gotman and Milner (1978) wrote that the finding of image-mediated verbal learning subserved by the right hemisphere 'had not been predicted' by the material-specificity model (p. 68).
} 
activation in studies by Golby et al. (2001), Kelley et al. (1998), and Simons et al. (2001), but whereas the Grady and Haxby studies have been cited as evidence against materialspecificity, these studies are interpreted as support for material-specificity. Unless the prediction of either format-specific or code-specific laterality is made explicit, experimental results are amenable to contradictory interpretations.

The initial neuroimaging studies that showed material-specific laterality in PFC used singly codable stimuli, as do many clinical tests for ipsilesional memory deficits. For singly codable stimuli, 'material type' (i.e. format) and mnemonic code covary. Subsequent neuroimaging studies have used dually codable stimuli, for which format and code are partially decoupled. These studies also claim to support material-specificity, but their results are difficult to interpret in terms of material type. Previously reviewed studies yielded the same result of bilateral (nonsignificantly lateralized) activation for a variety of stimulus materials: object pictures, complex scenes, some familiar faces, some unfamiliar faces, and some nouns. Do these items constitute a material type?

If material type modulates the hemispheric laterality of activation in PFC during memory processing, then reasonably consistent laterality should be found across studies that use a given stimulus type in similar tasks. This is not the case, not even when comparison is restricted to a particular category of stimuli within a material type. For example, some nouns have elicited left lateralized activation during encoding, while other nouns, which differ systematically in content but not in form, have elicited bilateral activation (Casasanto et al., 2001b; Friederici et al., 2000; Kounios \& Holcomb, 1994; Schmidt et al., 2002). Some unfamiliar faces have elicited preferential right hemisphere activation, others bilateral activation, and still others preferential left hemisphere activation (Casasanto et al., 2001a; Golby et al., 2001; Grady et al., 1995; Haxby et al., 1996; Kelley et al., 1998; McDermott et al., 1999). These variations are not random, rather they appear to reflect imageability and verbalizability, but they do not reflect any systematic variation in material type. Instead, these studies show systematic variation in hemispheric recruitment even though material type is held constant.

To summarize, material-specificity allows that hemispheric specialization may depend either on the external format of a stimulus or on the format-nonspecific internally generated codes by which it is represented in memory. Thus, material-specificity conflates two hypotheses regarding specialization at two different levels of mental representation: format-specificity and code-specificity. Many experiments using singly codable stimuli support both hypotheses, possibly because format and code covary. In contrast, numerous behavioral, neuropsychological, and neuroimaging experiments using dually codable stimuli disconfirm format-specificity and support code-specificity. Although 'material type' is ambiguous, 'material' is ordinarily construed as format, not code. Therefore, evidence in favor of code-specificity and against format-specific laterality calls for a reappraisal of the material-specific model as a principle of functional arealization in PFC.

Code-specificity has advantages over material-specificity as a working hypothesis to predict hemispheric effects in lateral posterior PFC: code-specificity is more inclusive of existing data, more specific, and has independently falsifiable components. As demonstrated previously, code-specificity accommodates data considered inconsistent with material-specificity (i.e. format-specificity). In principle, code-specificity can account for all hemispheric effects explained by format-specificity, but not the other 
way around, due to the asymmetric relationship between format and code described above. Whereas material-specificity is equivocal regarding the level of mental representation on which hemispheric specialization is hypothesized to depend, code-specificity predicts that laterality in certain PFC regions depends on mnemonic codes generated internally in response to both stimulus and task demands, not on bottom-up, input driven stimulus representations. Finally, whereas material-specificity posits hemispheric effects based on a single verbal/nonverbal dichotomy (or a single verbal/nonverbal continuum, as suggested by Golby et al., 2001), code-specificity proposes two independent continuums, one predicting activation in left hemisphere and the other in right hemisphere PFC structures. ${ }^{4}$ Thus, the verbalizability and imageability hypotheses that constitute code-specificity can be tested independently.

\section{Code-specificity and meaning}

The studies reviewed in Section 6 demonstrate how the mental representation of a single stimulus may be fractionated into verbal and nonverbal codes, which have differently lateralized neural substrates. The decomposition of a stimulus into separable mnemonic codes with discrete neural loci is controversial, but the analogous process of decomposing a stimulus into neurally separable perceptual components is uncontroversial (e.g. when we see a red vertical bar, the features 'red' and 'vertical' are instantiated separately in visual cortex). What is it about dual coding that raises some cognitive scientists' theoretical hackles?

Neuroimagers have appealed to Paivio's $(1971,1991)$ dual coding theory (DCT) to account for hemispheric effects during memory experiments, but have not specified which aspects of Paivio's theory obtain. In particular, the role of verbal and nonverbal codes in accessing (or constituting) the meaning of stimuli is unspecified. On Paivio's view, word meaning is stored in verbal representations, and picture meaning in imaginal representations. Abstract word meanings are represented only in the verbal system and nonnameable picture meanings only in the imaginal system, whereas the meanings of imageable words and nameable objects are represented twice, once in each system. Words provide direct access to verbal and 'abstract' semantics, but indirect access to imagistic semantics. Likewise, pictures provide direct access to imagistic semantics but indirect access to verbal and abstract semantics. Paivio (1991) posits semantic representations that are modality-specific and format-specific, and interprets hemispheric specialization for verbal and nonverbal memory as evidence in favor of DCT and against knowledge representations that may be equally accessible via different sensory modalities and surface formats. The claim of distinct (and presumably lateralized) verbal and imagistic semantics is central to Paivio's theory, so it must be assumed when neuroimaging researchers explain

\footnotetext{
${ }^{4}$ McDermott et al. (1999) also suggest that "verbal and nonverbal processing are not represented by a single continuum" (p. 637), but the authors do not suggest any continuums on which verbal and nonverbal processes might be represented, or what patterns of activation would be predicted to correlate with variations along these continuums.
} 
their results by making unqualified reference to DCT that they endorse Paivio's position on the relationship between format and meaning.

Neuroimaging results that support code-specificity are consistent with DCT, however they are equally consistent with an alternative developed by Potter et al., conceptual coding theory (CCT). As in DCT, CCT posits a structural distinction between the mnemonic representations of an object's name and the same object's form, however CCT also posits an additional mnemonic representation underlying them both, which is codenonspecific and modality-nonspecific (Potter, 1979). According to CCT, words, pictures, and direct percepts are all structurally distinct yet functionally equivalent 'way stations' along paths that converge on common knowledge representations. ${ }^{5}$

Previously reviewed neuroimaging studies may be agnostic with respect to the roles of verbal and nonverbal mnemonic codes in representing meaning, but other findings constrain their interpretation. Several studies show that regions of the left inferior PFC are more active during deep, meaning-based encoding than shallow structural encoding of verbal stimuli (Baker, Sanders, Macotta, \& Buckner, 2001; Casasanto et al., 2002; Fletcher et al., 2002; Kapur et al., 1994; Otten, Henson, \& Rugg, 2001). Other studies demonstrate specialization within LIPFC for lexical vs. semantic processing (for reviews, see Fiez, 1997; Wagner, 1999). Maintaining phonological codes preferentially engages the lateral posterior LIPFC (BA 6/44), and semantic retrieval the lateral anterior LIPFC (BA 45/47). Most studies that demonstrate form/meaning dissociations in LIPFC have used verbal materials. While neuroimaging studies have helped to distinguish the specific loci of lexical vs. semantic processing, it has long been known that some lexical and semantic processes are separable. Research on semantic access in fluent bilinguals (Illes et al., 1999; Potter, So, Von Eckardt, \& Feldman, 1984) shows that meaning is not preferentially associated with any particular lexical code, and a century of aphasia research shows that relatively normal semantic access can proceed in the absence of normal verbal processing. A novel finding from the neuroimaging literature, however, is that semantic retrieval appears to depend upon the same neural machinery in the LIPFC for both verbal and nonverbal stimuli.

Functional neuroimaging studies of conceptual priming suggest that access to semantic memory depends upon common retrieval processes localized in anterior LIPFC, regardless of the modality or format of the retrieval cue. Whereas perceptual priming is behavioral facilitation (e.g. a decrease in reaction time (RT)) resulting from the reprocessing of perceptual stimulus properties, conceptual priming is the behavioral facilitation that results from repeated access to semantic properties (Tulving \& Schacter, 1990). The neural correlate of semantic priming in PFC is the reduction of activation in regions that subserve semantic access upon repeated stimulus processing. Repeated semantic processing of words reduces activation in lateral anterior PFC, but repeated nonsemantic processing of words does not (Demb et al., 1995). This effect is not modality dependent, and has been demonstrated when verbal responses were cued visually and aurally (Buckner, Koutstaal, Schacter, \& Rosen, 2000). Behavioral and neural semantic priming is also independent of stimulus format. Wagner, Desmond, Demb, Glover, and Gabrieli

\footnotetext{
5 The nature of these underlying knowledge representations is a central problem in the cognitive sciences. What is important for the present discussion is that no such code-nonspecific representation is posited in DCT.
} 
(1997) showed RT facilitation and neural priming upon semantic reprocessing of both words and pictures in LIPFC but not RIPFC. This result is consistent with the earlier findings of LIPFC priming during repeated relative to initial picture naming (Blaxton, Gabrielli, Park, \& Figlozzi, 1997), and of a common left hemisphere network subserving semantic processing of words and pictures, which includes the anterior LIPFC (Vandenberghe, Price, Wise, Josephs, \& Frackowiak, 1996). These data are inconsistent with Paivio's claim of format-specific semantic access, and challenge the assumption that the semantics of words and pictures are distinct and differently lateralized.

What is the function of the proposed verbal and imaginal representational codes maintained by the left and right lateral posterior PFC? The preceding discussions rule out two possibilities. Hemispheric effects that are consistent with code-specificity but inconsistent with format-specificity mediate against the possibility that 'material-specific' regions of lateral posterior PFC subserve bottom-up, perceptually driven stimulus representations. The finding of conceptual priming for both words and pictures in anterior LIPFC mediates against a role for the differently lateralized verbal and imagistic codes in semantic access. Since posterior, not anterior cortical damage generally results in semantic memory deficits (McCarthy \& Warrington, 1988), there is no reason to suspect that lateral PFC supports long-term stored knowledge representations for verbal or nonverbal materials.

It has been suggested that inferior prefrontal activations observed during declarative memory tasks reflect the contribution of working memory processes to long-term encoding and retrieval (Buckner \& Koutstaal, 1998; Wagner, 1999). Regions of left and right lateral PFC that show code-specificity in declarative memory tasks overlap with regions implicated in maintaining working memory representations of phonological information and visuospatial information, respectively (Fiez, 1997; Smith et al., 1998; Smith \& Jonides, 1999). During intentional encoding into declarative memory, posterior lateral PFC activations may reflect volitional recruitment of verbal or visuospatial rehearsal operations. During incidental encoding tasks such as semantic judgment, goal-relevant information must be retrieved from semantic memory, possibly by anterior LIPFC mediated retrieval processes. In order for retrieved information to be evaluated it must be maintained online, engaging left or right lateral posterior PFC depending upon the type of information retrieved. During declarative memory retrieval, it may be necessary to maintain a working memory representation of the test probe in order to carry out retrieval search, and to maintain the retrieved information online in order to decide on a response (Wagner, 1999). Available data are consistent with the hypothesis that code-specific activations in PFC during declarative memory tasks reflect differently lateralized verbal and visuospatial working memory representations.

Material-specificity has been thought to predict stimulus-related hemispheric variations, all other things being equal. In practice, hemispheric effects in lateral PFC are modulated not only by stimulus parameters but also by task parameters such as trial duration and orienting task, which bias strategies used during encoding or retrieval and affect depth or success of encoding (Baker et al., 2001; Brewer, Zhao, Desmond, Glover, \& Gabrieli, 1998; Casasanto et al., 2002; Fletcher et al., 2002; Kapur et al., 1994; Otten et al., 2001; Wagner et al., 1998b). On a material-specific account, it seems that multiple principles are needed to explain hemispheric effects in lateral posterior 
PFC. Since the format of a stimulus does not change with variations in task parameters, it has been posited that 'task-specificity' interacts with material-specificity, and that task-related hemispheric effects sometimes override stimulus-related effects. Codespecificity may provide a more parsimonious alternative. Although the perceptual format of a given stimulus is task-invariant, the representational codes invoked by the stimulus are task-dependent. This has been demonstrated in a recent neuroimaging experiment that manipulated levels of processing (Craik \& Lockhart, 1972). Right PFC showed greater activity during verbal learning when words were encoded based on structural features (i.e. presence or absence of repeated letters) rather than on meaning (Fletcher et al., 2002). The authors attribute this difference to the greater requirement during the structural task to maintain and manipulate visuospatial representations. It may be possible to demonstrate a similar effect within a single level of processing. For example, different semantic judgments performed on a given object name may yield differently lateralized activation, even when stimulus presentation and response parameters are controlled (e.g. the two judgments are matched for difficulty and RT). Judging whether the referent has more than four corners may elicit greater right PFC activation than judging whether it existed before the 20th century. Both judgments require accessing and maintaining stored knowledge about the object, but the relevant subsets of knowledge differ across tasks, as does the extent to which visuospatial working memory processes are likely to be engaged. If activation in posterior lateral PFC is code-specific, and the verbal and imaginal 'codes' are working memory representations, then code-specificity may account for both stimulus- and task-related hemispheric effects.

\section{Conclusions}

In prefrontal regions implicated in material-specific mnemonic processing, patterns of hemispheric specialization shown previously across material types have been demonstrated within verbal and nonverbal material types. Functional neuroimaging studies show that left lateral prefrontal activation correlates with the verbalizability of nonverbal stimuli, and right lateral prefrontal regions with the imageability of verbal stimuli. These results call into question the extent to which hemispheric specialization in the PFC depends on the external characteristics of a stimulus as opposed to internally generated stimulus representations, and whether material-specificity can account for neuroimaging results. It is argued that material-specificity does not specify the level of mental representation at which it has been hypothesized to operate, and it is therefore difficult to falsify. Material-specificity is analyzed into two hypotheses, one predicting hemispheric specialization based on stimulus format, and the other on format-nonspecific mnemonic codes. Data reviewed show that when format and code are in opposition, numerous studies disconfirm format-specificity and support codespecificity.

Code-specificity comprises two independently falsifiable hypotheses concerning hemispheric effects of verbalizability and imageability, both of which are supported by behavioral and lesion-deficit data, as well as many (but not all) of the neuroimaging studies 
reviewed. Although many of the findings that support code-specificity also support Paivio's DCT (and vice-versa), the theoretical commitments of code-specificity can be divorced from those of DCT. In particular, code-specific activation does not imply differently lateralized verbal and imagistic semantics. It is hypothesized that the verbal and imagistic codes responsible for hemispheric effects in lateral PFC during declarative memory tasks are in fact working memory representations, and constitute an intermediate level of information processing, between format-specific perceptual processing and format-nonspecific semantic memory processing.

It is possible that both format-specific and code-specific regions exist in prefrontal cortex, and that code-specific activity may recapitulate perceptually driven format-specific activity to some extent, analogous to the reactivation of posterior cortical areas involved in visual perception during visual imagery (Kosslyn, Behrmann, \& Jeannerod, 1995). Testing these possibilities requires varying the surface format of stimuli and the representational codes they invoke.

\section{Acknowledgements}

Many thanks to Molly Potter and Anthony Wagner for challenging and enlightening discussions, and for comments on an earlier draft of this article. This work was supported by an NSF graduate research fellowship to the author.

\section{References}

Baker, J. T., Sanders, A. L., Macotta, L., \& Buckner, R. L. (2001). Neural correlates of verbal memory encoding during semantic and structural processing tasks. Neuroreport, 10(10), 1-7.

Beauregard, M., Chertkow, H., Bub, D., Murtha, S., Dixon, R., \& Evans, A. (1997). The neural substrate for concrete, abstract, and emotional word lexica: a positron emission tomography study. Journal of Cognitive Neuroscience, 9(4), 441-461.

Blakemore, C. B., \& Falconer, M. A. (1967). Long-term effects of anterior temporal lobectomy on certain cognitive functions. Journal of Neurology, Neurosurgery, and Psychiatry, 30(4), 364-367.

Blaxton, T. A., Gabrielli, J. D. E., Park, S., \& Figlozzi, C. M. (1997). Conceptual and perceptual transfer in implicit memory: A PET activation study of primed picture naming. Unpublished manuscript.

Bower, G. (1970). Imagery as a relational organizer in associative learning. Journal of Verbal Learning and Verbal Behavior, 9(5), 529-533.

Brewer, J. B., Zhao, Z., Desmond, J. E., Glover, G. H., \& Gabrieli, J. D. (1998). Making memories: brain activity that predicts how well visual experience will be remembered. Science, 281(5380), 1185-1187.

Buckner, R. L., \& Koutstaal, W. (1998). Functional neuroimaging studies of encoding, priming, and explicit memory retrieval. Proceedings of the National Academy of Science USA, 95(3), 891-898.

Buckner, R. L., Koutstaal, W., Schacter, D. L., \& Rosen, B. R. (2000). Functional MRI evidence for a role of frontal and inferior temporal cortex in amodal components of priming. Brain, 123(Pt 3), 620-640.

Casasanto, D., Kounios, J., \& Detre, J (2001). Hemispheric effects of concreteness in pictures and words. Paper presented at the Cognitive Science Society, Edinburgh, UK.

Casasanto, D., Kounios, J., Roc, A., Mintzer, D., Maldjian, J., \& Detre, J. (2001b). Hemispheric effects of concreteness during verbal episodic memory encoding. Neuroimage, 13, S644.

Casasanto, D. J., Killgore, W. D., Maldjian, J. A., Glosser, G., Alsop, D. C., Cooke, A. M., Grossman, M., \& Detre, J. A. (2002). Neural correlates of successful and unsuccessful verbal memory encoding. Brain and Language, 80(3), 287-295. 
Chertkow et al., in press. See Beauregard et al., 1997.

Corkin, S. (1965). Tactually-guided maze learning in man: effects of unilateral cortical excisions and bilateral hippocampal lesions. Neuropsychologia, 3, 339-351.

Craik, F. I. M., \& Lockhart, R. S. (1972). Levels of processing: a framework for memory research. Journal of Verbal Learning and Verbal Behavior, 11, 671-684.

Demb, J. B., Desmond, J. E., Wagner, A. D., Vaidya, C. J., Glover, G. H., \& Gabrieli, J. D. (1995). Semantic encoding and retrieval in the left inferior prefrontal cortex: a functional MRI study of task difficulty and process specificity. Journal of Neuroscience, 15(9), 5870-5878.

Fiez, J. A. (1997). Phonology, semantics, and the role of the left inferior prefrontal cortex. Human Brain Mapping, 5(2), 79-83.

Fletcher, P. C., Palomero-Gallagher, N., Zafiris, O., Fink, G. R., Tyler, L. K., \& Zilles, K. (2002). The influence of explicit instructions and stimulus material on lateral frontal responses to an encoding task. Neuroimage, 17(2), $780-791$.

Fletcher, P. C., Shallice, T., Frith, C. D., Frackowiak, R. S., \& Dolan, R. J. (1996). Brain activity during memory retrieval. The influence of imagery and semantic cueing. Brain, 119(Pt 5), 1587-1596.

Friederici, A. D., Opitz, B., \& von Cramon, D. Y. (2000). Segregating semantic and syntactic aspects of processing in the human brain: an fMRI investigation of different word types. Cerebral Cortex, 10(7), $698-705$.

Golby, A. J., Poldrack, R. A., Brewer, J. B., Spencer, D., Desmond, J. E., Aron, A. P., \& Gabrieli, J. D. (2001). Material-specific lateralization in the medial temporal lobe and prefrontal cortex during memory encoding. Brain, 124(Pt 9), 1841-1854.

Goldman-Rakic, P. (2000). Localization of function all over again. Neuroimage, 11, 451-457.

Gorno-Tempini, M. L., Price, C. J., Josephs, O., Vandenberghe, R., Cappa, S. F., Kapur, N., Frackowiak, R. S., \& Tempini, M. L. (1998). The neural systems sustaining face and proper-name processing. Brain, 121(Pt 11), 2103-2118

Grady, C. L., McIntosh, A. R., Horwitz, B., Maisog, J. M., Ungerleider, L. G., Mentis, M. J., Pietrini, P., Schapiro, M. B., \& Haxby, J. V. (1995). Age-related reductions in human recognition memory due to impaired encoding. Science, 269(5221), 218-221.

Haxby, J. V., Ungerleider, L. G., Horwitz, B., Maisog, J. M., Rapoport, S. I., \& Grady, C. L. (1996). Face encoding and recognition in the human brain. Proceedings of the National Academy of Science USA, 93(2), 922-927.

Illes, J., Francis, W. S., Desmond, J. E., Gabrieli, J. D., Glover, G. H., Poldrack, R., Lee, C. J., \& Wagner, A. D. (1999). Convergent cortical representation of semantic processing in bilinguals. Brain and Language, 70(3), 347-363.

Jones, M. K. (1974). Imagery as a mnemonic aid after left temporal lobectomy: contrast between material-specific and generalized memory disorders. Neuropsychologia, 12(1), 21-30.

Jones-Gotman, M. (1986). Memory for designs: the hippocampal contribution. Neuropsychologia, 24(2), 193-203.

Jones-Gotman, M., \& Milner, B. (1978). Right temporal-lobe contribution to image-mediated verbal learning. Neuropsychologia, 16(1), 61-71.

Kaplan, R. F., Meadows, M. E., Verfaellie, M., Kwan, E., Ehrenberg, B. L., Bromfield, E. B., \& Cohen, R. A. (1994). Lateralization of memory for the visual attributes of objects: evidence from the posterior cerebral artery amobarbital test. Neurology, 44(6), 1069-1073.

Kapur, S., Craik, F. I., Tulving, E., Wilson, A. A., Houle, S., \& Brown, G. M. (1994). Neuroanatomical correlates of encoding in episodic memory: levels of processing effect [see comments]. Proceedings of the National Academy of Science USA, 91(6), 2008-2011.

Kelley, W., Buckner, R., Miezin, F., Cohen, N., Raichle, M., \& Petersen, S. (1999). Encoding of famous and nonfamous faces using fMRI. Society for neurosciences abstracts (1999)

Kelley, W. M., Miezin, F. M., McDermott, K. B., Buckner, R. L., Raichle, M. E., Cohen, N. J., Ollinger, J. M., Akbudak, E., Conturo, T. E., Snyder, A.Z., \& Petersen, S. E. (1998). Hemispheric specialization in human dorsal frontal cortex and medial temporal lobe for verbal and non verbal memory encoding. Neuron., 20(5), 927-936 
Kelley, W. M., Ojemann, J. G., Wetzel, R. D., Derdeyn, C. P., Moran, C. J., Cross, D. T., Dowling, J. L., Miller, J. W., \& Petersen, S. E. (2002). Wada testing reveals frontal lateralization for the memorization of words and faces. Journal of Cognitive Neuroscience, 14(1), 116-125.

Kiehl, K. A., Liddle, P. F., Smith, A. M., Mendrek, A., Forster, B. B., \& Hare, R. D. (1999). Neural pathways involved in the processing of concrete and abstract words. Human Brain Mapping, 7(4), $225-233$.

Kirchhoff, B. A., Wagner, A. D., Maril, A., \& Stern, C. E. (2000). Prefrontal-temporal circuitry for episodic encoding and subsequent memory. Journal of Neuroscience, 20(16), 6173-6180.

Kosslyn, S. M., Behrmann, M., \& Jeannerod, M. (1995). The cognitive neuroscience of mental imagery. Neuropsychologia, 33(11), 1335-1344.

Kounios, J., \& Holcomb, P. J. (1994). Concreteness effects in semantic processing: ERP evidence supporting dual-coding theory. Journal of Experimental Psychology. Learning, Memory, and Cognition, 20(4), $804-823$.

Lakoff, G., \& Johnson, M. (1980). Metaphors we live by. Chicago: University of Chicago Press.

Leveroni, C. L., Seidenberg, M., Mayer, A. R., Mead, L. A., Binder, J. R., \& Rao, S. M. (2000). Neural systems underlying the recognition of familiar and newly learned faces. Journal of Neuroscience, 20(2), 878-886.

Martin, A. (1999). Automatic activation of the medial temporal lobe during encoding: lateralized influences of meaning and novelty. Hippocampus, 9(1), 62-70.

McCarthy, R. A., \& Warrington, E. K. (1988). Evidence for modality-specific meaning systems in the brain. Nature, 334(6181), 428-430.

McDermott, K. B., Buckner, R. L., Petersen, S. E., Kelley, W. M., \& Sanders, A. L. (1999). Set- and code-specific activation in frontal cortex: an fMRI study of encoding and retrieval of faces and words. Journal of Cognitive Neuroscience, 11(6), 631-640.

Miller, E. K., \& Cohen, J. D. (2001). An integrative theory of prefrontal cortex function. Annual Review of Neuroscience, 24, 167-202.

Milner, B. (1968). Visual recognition and recall after right temporal lobe excision in man. Neuropsychologia, 6, 191-209.

Milner, B. (1974). Hemispheric specialization: scope and limits. In B. Milner (Ed.), Hemispheric specialization and interaction. Cambridge: MIT Press.

Milner, B. (1982). Some effects of frontal-lobe lesions in man. Philosophical Transactions of the Royal Society of London, 298, 211-226.

Milner, B., \& Petrides, M. (1984). Behavioral effects of frontal lobe lesions in man. Trends in Neurosciences, 403-407.

Nyberg, L., Cabeza, R., \& Tulving, E. (1996). PET studies of encoding and retrieval: The Hera model. Psychonomic Bulletin and Review, 3(2), 135-148.

Nystrom, L. E., Braver, T. S., Sabb, F. W., Delgado, M. R., Noll, D. C., \& Cohen, J. D. (2000). Working memory for letters, shapes, and locations: fMRI evidence against stimulus-based regional organization in human prefrontal cortex. Neuroimage, 11(5 Pt 1), 424-446.

Ojemann, G. A., \& Dodrill, C. B. (1985). Verbal memory deficits after left temporal lobectomy for epilepsy. Mechanism and intraoperative prediction. Journal of Neurosurgery, 62(1), 101-107.

Otten, L. J., Henson, R. N., \& Rugg, M. D. (2001). Depth of processing effects on neural correlates of memory encoding: relationship between findings from across- and within-task comparisons. Brain, 124(Pt 2), 399-412.

Owen, A., Stern, S., Look, R., Tracey, I., Rosen, B., \& Petrides, M. (1998). Functional organization of spatial and nonspatial working memory processing within the human lateral frontal cortex. Proc. Natl. Acad. Sci., 95, $7721-7726$.

Paivio, A. (1971). Imagery and verbal processes. New York: Hold, Rinehart and Winston.

Paivio, A. (1991). Dual coding theory: retrospect and current status. Canadian Journal of Psychology, 45(3), $255-287$.

Paivio, A., \& Csapo, K. (1969). Concrete image and verbal memory codes. Journal of Experimental Psychology, $80(2), 279-285$.

Petrides, M. (1985). Deficits on conditional associative-learning tasks after frontal- and temporal-lobe lesions in man. Neuropsychologia, 23(5), 601-614. 
Petrides, M. (1994). The frontal lobes and behaviour. Current Opinion in Neurobiology, 4, 207-211.

Plenger, P. M., Breier, J. I., Wheless, J. W., Ridley, T. D., Papanicolaou, A. C., Brookshire, B., Thomas, A., Curtis, V., \& Willmore, L. J. (1996). Lateralization of memory for music: evidence from the intracarotid sodium amobarbital procedure. Neuropsychologia, 34(10), 1015-1018.

Postle, B. R., Stern, C. E., Rosen, B. R., \& Corkin, S. (2000). An fMRI investigation of cortical contributions to spatial and nonspatial visual working memory. Neuroimage, 11(5 Pt 1), 409-423.

Potter, M. C. (1979). Mundane symbolism: The relations among objects, names, and ideas. In N. Smith, \& M Franklin (Eds.), Symbolic functioning in childhood (pp. 41-65). Hillsdale: L. Erlbaum.

Potter, M., So, K., Von Eckardt, B., \& Feldman, L. (1984). Lexical and conceptual representation in beginning and proficient bilinguals. Journal of Verbal Learning and Verbal Behavior, 23, 23-38.

Saffran, E., \& Sholl, A. (2000). Clues to the functional and neural architecture of word meaning. In C. Brown, \& P. Hagoort (Eds.), The Neurocognition of Language (pp. 241-272). Oxford: Oxford University Press.

Samson, S., \& Zatorre, R. J. (1991). Recognition memory for text and melody of songs after unilateral temporal lobe lesion: evidence for dual encoding. Journal of Experimental Psychology. Learning Memory, and Cognition, 17(4), 793-804.

Schmidt, D., Krause, B. J., Mottaghy, F. M., Halsband, U., Herzog, H., Tellmann, L., \& Muller-Gartner, H. W. (2002). Brain systems engaged in encoding and retrieval of word-pair associates independent of their imagery content or presentation modalities. Neuropsychologia, 40(4), 457-470.

Seamon, J., \& Gazzaniga, M. (1973). Coding strategies and cerebral laterality effects. Cognitive Psychology, 5, $249-256$.

Seidenberg, M., Griffith, R., Sabsevitz, D., Moran, M., Haltiner, A., Bell, B., Swanson, S., Hammeke, T., \& Hermann, B. (2002). Recognition and identification of famous faces in patients with unilateral temporal lobe epilepsy. Neuropsychologia, 40(4), 446-456.

Shimamura, A. P. (1995). Memory and the prefrontal cortex. Annals of the New York Academy of Sciences, 769, $151-159$.

Simons, J. S., Graham, K. S., Owen, A. M., Patterson, K., \& Hodges, J. R. (2001). Perceptual and semantic components of memory for objects and faces: a pet study. Journal of Cognitive Neuroscience, 13(4), 430-443.

Smith, E. E., \& Jonides, J. (1999). Storage and executive processes in the frontal lobes. Science, 283(5408), $1657-1661$

Smith, E. E., Jonides, J., Marshuetz, C., \& Koeppe, R. A. (1998). Components of verbal working memory: evidence from neuroimaging. Proceedings of the National Academy of Science USA, 95(3), 876-882.

Tulving, E., Kapur, S., Craik, F. I., Moscovitch, M., \& Houle, S. (1994). Hemispheric encoding/retrieval asymmetry in episodic memory: positron emission tomography findings [see comments]. Proceedings of the National Academy of Science USA, 91(6), 2016-2020.

Tulving, E., Markowitsch, H. J., Craik, F. E., Habib, R., \& Houle, S. (1996). Novelty and familiarity activations in PET studies of memory encoding and retrieval. Cerebral Cortex, 6(1), 71-79.

Tulving, E., \& Schacter, D. L. (1990). Priming and human memory systems. Science, 247(4940), 301-306.

Vandenberghe, R., Price, C., Wise, R., Josephs, O., \& Frackowiak, R. S. (1996). Functional anatomy of a common semantic system for words and pictures. Nature, 383(6597), 254-256.

Wagner, A. D., Desmond, J. E., Demb, J. B., Glover, G. H., \& Gabrieli, J. D. E. (1997). Semantic repetition priming for verbal and pictorial knowledge: a functional MRI study of left inferior prefrontal cortex. Journal of Cognitive neuroscience, 9(6), 714-726.

Wagner, A. D. (1999). Working memory contributions to human learning and remembering. Neuron, 22(1), $19-22$.

Wagner, A. D., Koutstaal, W., Maril, A., Schacter, D. L., \& Buckner, R. L. (2000). Task-specific repetition priming in left inferior prefrontal cortex. Cerebral Cortex, 10(12), 1176-1184.

Wagner, A. D., Poldrack, R. A., Eldridge, L. L., Desmond, J. E., Glover, G. H., \& Gabrieli, J. D. (1998a). Material-specific lateralization of prefrontal activation during episodic encoding and retrieval. Neuroreport, 9(16), 3711-3717.

Wagner, A. D., Schacter, D. L., Rotte, M., Koutstaal, W., Maril, A., Dale, A. M., Rosen, B. R., \& Buckner, R. L. (1998b). Building memories: remembering and forgetting of verbal experiences as predicted by brain activity [see comments]. Science, 281(5380), 1188-1191. 
Warrington, E., \& Shallice, T. (1984). Category specific brain impairments. Brain, (107), 829-854.

Wheeler, M. A., Stuss, D. T., \& Tulving, E. (1995). Frontal lobe damage produces episodic memory impairment. Journal of International Neuropsychological Society, 1(6), 525-536.

Whitehouse, P. J. (1981). Imagery and verbal encoding in left and right hemisphere damaged patients. Brain and Language, 14(2), 315-332.

Zatorre, R. J., Mondor, T. A., \& Evans, A. C. (1999). Auditory attention to space and frequency activates similar cerebral systems. Neuroimage, 10(5), 544-554. 